\title{
A Note on Universal Black holes of a Dynamic System
}

\author{
Kondru Prasen ${ }^{1}$ Prof. Dr. K. Raja Rama Gandhi ${ }^{2}$
}

12th Grade, Sri Chaitanya IIT Academy, Ramnagar, Visakhapatnam ${ }^{1}$

Resource person in Math for Oxford University Press and Professor in Math at BITS-Vizag ${ }^{2}$

\section{E-mail: rrmath28@gmail.com}

\begin{abstract}
We know that while considering a mathematical dynamical system by assigning a rule of cubing the individual digits of a particular number continuously, we will end up at some number which is recurring and can be called as the Universal Black hole of that particular system. However, some numbers only end at 153 . The reasons for this occurrence will be discussed by a theorem. Also, we list such numbers which are $<5000$.
\end{abstract}

\section{Introduction}

I'm very fascinated about the Theory of Numbers to which I have been introduced to by Prof. Dr. K. Raja Rama Gandhi [4] two years ago. This resulted in an innate love for that field and 3 research papers which have been published in Asian Journal of Mathematics and Physics - ISSN 2308-3131, titled: Generalization of Concatenation Numbers [1], Generalization of Concatenation Numbers- 2 [2] and A Conjecture about Functions [3]. Those 3 papers attained a huge response and in turn have motivated me to learn more about the Theory of Numbers and also write a good number of research papers.

Interestingly, during the month of December 2013, the DST collaborated with Andhra University and organized a program called INSPIRE in which professors from different fields gave lectures about different fields of Science. I had to eagerly wait for the fifth day, which was assigned to Mathematics, because I was personally interested to listen to lectures about Number Theory. Although there was no lecture specifically about Number Theory, the first lecture of the day, which discussed about Mathematical Dynamical Systems gave me an idea which led to this paper.

In the lecture, the Professor took a dynamical system with the above stated rule and applied it to the number 21 which resulted in an output of 153 as shown below.

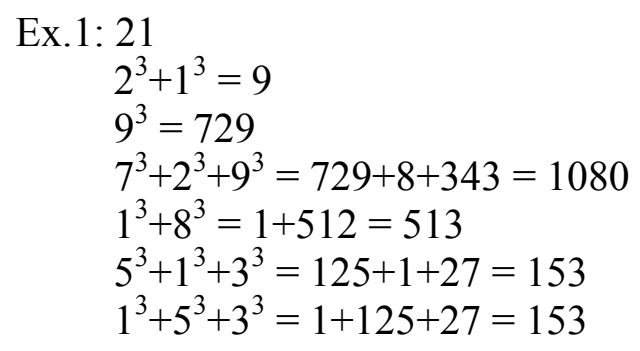

As soon as I returned home, I applied the same rule to two random numbers 12 and 13 as shown below.
Ex.2: 12
$1^{3}+2^{3}=9$
$9^{3}=729$




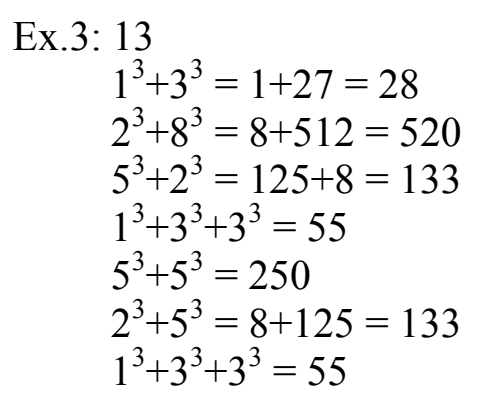

As you can see, in examples 1 and 2, the number 153 is the recurring output while in example 3 , 133 is the recurring number in a loop. The reasons for this including the theorem will be discussed in the next section.

\section{Observation(s) and Theorem(s)}

Interestingly, the first two examples are divisible by 3 . However, the number 13 isn't divisible by 3 . Finally, I checked this for a lot of random numbers which are divisible by 3 and all these numbers ended up at 153. After this, my curiosity grew as to why this property is being observed by multiples of 3 and after a deep study I got to know that 153 is a number which has an immense importance in the Bible [5].

Not only this, but 153 is also a special number from my perspective as it can be written as:

$$
\begin{aligned}
153 & =1^{3}+5^{3}+3^{3} \\
& =1 !+2 !+3 !+4 !+5 ! \\
& =100+28+25
\end{aligned}
$$

where 100 is a square number, 28 is a triangular number and 25 is a circular number.

The following observations and theorems will conclude the reasons of occurrence of 153 when a number is divisible by 3 .

Observation \#1: $\mathrm{f}: \mathrm{N} \rightarrow \mathrm{N}$

$$
\text { a1 .....ad } \rightarrow \mathrm{a}^{3}+\ldots . .+\mathrm{a}_{\mathrm{d}}^{3}
$$

At the highest value for a d-digit number is obtained for $10^{d}-1$ and as $f(9999)=4\left(9^{3}\right) \leqslant 9999$.

Theorem \#1: $\mathrm{f}(\mathrm{n}) \leq \mathrm{n}$ is $(\downarrow)$ for $\mathrm{n} \geq 10^{4}$, and also $\mathrm{f}(\mathrm{n})<10^{4}$ for $\mathrm{n} \leq 10^{4}$.

Proof: For $\mathrm{n} \geqslant 10^{4}-1$, we have $\mathrm{d} \geqslant 5$.

Thus, $\mathrm{f}(\mathrm{n}) \leqslant \mathrm{f}\left(10^{\mathrm{d}+1}-1\right)$.

But $f\left(10^{d+1}-1\right)=f(9 \ldots .99) \leqslant 10^{d}-1$, since $d>4$.

Theorem \#2: For any $n \in N$, there is $k \in N$, such that $f^{k}(n)=153$ if $n$ is divisible by 3 .

Proof: If a number multiple of 3 , when $\mathrm{a}^{3} \equiv \mathrm{a}(\bmod 3)$

$3 \mid 153$ or $3 \mid 1+5+3=9$.

In general, $9^{3}<\hat{1000}$.

$\mathrm{S}(\mathrm{n})<\mathrm{n}$, when $\mathrm{n}>10000$

and $\mathrm{S}(\mathrm{n})<10000$, when $\mathrm{n} \leqslant 10000$. (These are not sharp bounds) 
Let us define the sequence $\left\{\mathrm{S}^{\mathrm{k}}(\mathrm{n})\right\}_{\mathrm{k} \geq 0}$ will be decreasing until it falls below 10000. Because of that, the function will eventually set in a finite loop. Thus, there is an ' 1 ' such that, for a sufficient large $K, s^{K+1}(n)=s^{k}(n)$ It is possible to calculate all fix points $n$ of $s$ for which $S(n)=n$. This applies only to 153 .

Observation \#2: The other fix points (I assume that) 370, 371 and 407, and of course 0 and 1, but these numbers are trivial. As we can see, none of these numbers are divisible by 3 , so they can only end up in 153 or in a cycle, but there aren't any cycles apparently. The only number that may be of interest is 11 , because a number in multiple of 11 , when the difference between alternating sum is divisible by 11 . Problem is that, $2^{3}=8 \equiv \pm 2(\bmod 11)$, divisibility is not conserved. For any other interesting numbers, we can search (by congruencies [6]) for $n$ such that,

$$
\begin{gathered}
\mathrm{i}^{3} \equiv \pm \mathrm{i}(\bmod \mathrm{n}) \forall 1 \leqslant \mathrm{i} \leqslant \mathrm{n} . \\
\text { Thus, } 2^{3}=8 \equiv \pm 2(\bmod \mathrm{n}) \rightarrow(1)
\end{gathered}
$$

(1) is true for $\mathrm{n}=2, \mathrm{n}=3, \mathrm{n}=5, \mathrm{n}=6$ and $\mathrm{n}=10$.

$\mathrm{n}=2$ does not work, because $\mathrm{S}(12)=1+8=9$ and 9 is odd, same as $\mathrm{n}=10$.

$$
\mathrm{S}(10)=1+0=1
$$

For $\mathrm{n}=6$, we get $\mathrm{S}(12)=9$.

For $\mathrm{n}=5$, we get $\mathrm{S}(10)=1$.

So this won't work either.

$\therefore$ Only multiples of 3 have the property that they are still multiple of 3 , after applying s.

\section{Appendix}

I observed that the following numbers which are $<5000$ will have the same behavior in the above stated dynamical system.

\section{$1=$}

$[1,10,100,112,121,211,778,787,877,1000,1012,1021,1102,1120,1189,1198,1201,1210,1234,1243,13$ $24,1342,1423,1432,1579,1597,1759,1795,1819,1891,1918,1957,1975,1981,201$, $2101,2110,2134,2143,2314,2341,2413,2431,2779,2797,2977,3124,3142,3214,3241,3412,3421,412$ $3,4132,4213,4231,4312,4321]$

\section{$250=$}

$[4,13,25,28,31,40,46,52,64,82,103,130,205,208,250,256,265,280,289,298,301,310,349,394,400,406$ ,439,448,460,484,493,502,520,526,562,589,598,604,625,640,652,802,820,829,844,859,892,895,92 $8,934,943,958,982,985,1003,1030,1111,1144,1222,1246,1249,1264,1294,1300,1333,1348,1366,138$ $4,1414,1426,1429,1438,1441,1456,1462,1465,1483,1492,1546,1564,1624,1636,1642,1645,1654,16$ $63,1777,1834,1843,1924,1942,2005,2008,2050,2056,2065,2080,2089,2098,2122,2146,2149,2164,2$ $194,2212,2221,2245,2254,2257,2266,2275,2416,2419,2425,2452,2461,2479,2491,2497,2500,2506$, $2524,2527,2542,2560,2572,2605,2614,2626,2641,2650,2662,2689,2698,2725,2749,2752,2794,280$ $0,2809,2869,2890,2896,2908,2914,2941,2947,2968,2974,2980,2986,3001,3010,3049,3094,3100,31$ $33,3148,3166,3184,3313,3331,3409,3418,3445,3454,3481,3490,3544,3556,3565,3616,3655,3661,3$ $667,3676,3766,3814,3841,3904,3940,4000,4006,4039,4048,4060,4084,4093,4114,4126,4129,4138$, $4141,4156,4162,4165,4183,4192,4216,4219,4225,4252,4261,4279,4291,4297,4309,4318,4345,435$ $4,4381,4390,4408,4411,4435,4444,4453,4459,4480,4495,4516,4522,4534,4543,4549,4555,4561,45$ 
$94,4600,4612,4615,4621,4651,4729,4792,4804,4813,4831,4840,4903,4912,4921,4927,4930,4945,4$ 954,4972]

\section{$370=$}

$[7,19,34,37,43,58,67,70,73,76,85,88,91,109,118,124,139,142,145,148,154,157,166,169,175,178,18$ $1,184,187,190,193,196,214,223,226,232,241,247,259,262,268,274,277,286,295,304,307,319,322,3$ $34,340,343,346,355,358,364,367,370,376,385,391,403,412,415,418,421,427,430,433,436,451,463$, $466,469,472,481,496,499,508,514,517,529,535,538,541,553,556,559,565,568,571,577,580,583,586$ ,592,595,607,616,619,622,628,634,637,643,646,649,655,658,661,664,667,670,673,676,682,685,68 $8,691,694,700,703,706,715,718,724,727,730,736,742,751,757,760,763,766,772,775,781,799,805,8$ $08,811,814,817,826,835,841,850,853,856,862,865,868,871,880,886,889,898,901,910,913,916,925$, $931,946,949,952,955,961,964,979,988,994,997,1009,1018,1024,1039,1042,1045,1048,1054,1057,1$ 066,1069,1075,1078,1081,1084,1087,1090,1093,1096,1108,1114,1117,1123,1126,1132,1135,1138,1 $141,1153,1156,1159,1162,1165,1168,1171,1177,1180,1183,1186,1195,1204,1213,1216,1225,1228,1$ $231,1240,1252,1255,1258,1261,1267,1276,1279,1282,1285,1297,130$

$9,1312,1315,1318,1321,1351,1357,1375,1381,1390,1402,1405,1408,1411,1420,1444,1447,1450,14$ $74,1477,1480,1504,1507,1513,1516,1519,1522,1525,1528,1531,1537,1540,1552,1555,1558,1561,1$ $567,1570,1573,1576,1582,1585,1591,1606,1609,1612,1615,1618,1621,1627,1651,1657,1660,1666$, $1669,1672,1675,1681,1690,1696,1699,1705,1708,1711,1717,1726,1729,1735,1744,1747,1750,175$ $3,1756,1762,1765,1771,1774,1780,1789,1792,1798,1801,1804,1807,1810,1813,1816,1822,1825,18$ $31,1840,1852,1855,1861,1870,1879,1888,1897,1900,1903,1906,1915,1927,1930,1951,196$ $0,1966,1969,1972,1978,1987,1996,2014,2023,2026,2032,2041,2047,2059,2062,2068,2$ $074,2077,2086,2095,2104,2113,2116,2125,2128,2131,2140,2152,2155,2158,2161,2167,2176,2179$, $2182,2185,2197,2203,2206,2215,2218,2224,2227,2230,2233,2236,2239,2242,2248,2251,2260,226$ $3,2269,2272,2278,2281,2284,2287,2293,2296,2299,2302,2311,2320,2323,2326,2329,2332,2335,23$ $53,2356,2359,2362,2365,2368,2386,2389,2392,2395,2398,2401,2407,2410,2422,2428,2449,2455,2$ $470,2482,2488,2494,2509,2512,2515,2518,2521,2533,2536,2539,2545,2551,2554,2563,2566,2569$, $2578,2581,2587,2590,2593,2596,2599,2602,2608,2611,2617,2620,2623,2629,2632,2635,2638,265$ $3,2656,2659,2665,2668,2671,2677,2680,2683,2686,2692,2695,2704,2707,2716,2719,2722,2728,27$ $40,2758,2761,2767,2770,2776,2782,2785,2788,2791,2806,2812,2815,2821,2824,282$

$7,2836,2839,2842,2848,2851,2857,2860,2863,2866,2872,2875,2878,2884,2887,2893,2$

$899,2905,2917,2923,2926,2929,2932,2935,2938,2944,2950,2953,2956,2959,2962,2965,2971,2983$, 2989,2992,2995,2998,3004,3007,3019,3022,3034,3040,3043,3046,3055,3058,3064,3067,3070,307 $6,3085,3091,3109,3112,3115,3118,3121,3151,3157,3175,3181,3190,3202,3211,32202,3034,3040,3$ 043,3046,3055,3058,3064,3067,3070,3076,3085,3091,3109,3112,3115,3118,3121,3151,3157,3175, $3181,3190,3202,3211,3220,3223,3226,3229,3232,3235,3253,3256,3259,3262,3265,3268,3286,328$ $9,3292,3295,3298,3304,3322,3325,3334,3340,3343,3346,3352,3355,3358,3364,3379,3385,3388,33$ $97,3400,3403,3406,3430,3433,3436,3448,3457,3460,3463,3475,3478,3484,3487,3505,3508,3511,3$ $517,3523,3526,3529,3532,3535,3538,3547,3550,3553,3562,3568,3571,3574,3580,3583,3586,3592$, $3604,3607,3622,3625,3628,3634,3640,3643,3652,3658,3670,3679,3682,3685,3688,3697,3700,370$ $6,3715,3739,3745,3748,375$

$1,3754,3760,3769,3778,3784,3787,3793,3796,3805,3811,3826,3829,3835,3838,3844,3$ $847,3850,3853,3856,3862,3865,3868,3874,3877,3883,3886,3889,3892,3898,3901,3910,3922,3925$, $3928,3937,3952,3967,3973,3976,3982,3988,4003,4012,4015,4018,4021,4027,4030,4033,4036,405$ $1,4063,4066,4069,4072,4081,4096,4099,4102,4105,4108,4111,4120,4144,4147,4150,4174,4177,41$ $80,4201,4207,4210,4222,4228,4249,4255,4270,4282,4288,4294,4300,4303,4306,4330,4333,4336,4$ $348,4357,4360,4363,4375,4378,4384,4387,4414,4417,4429,4438,4441,4447,4456,4465,4468,4471$, $4474,4477,4483,4486,4492,4501,4510,4525,4537,4546,4552,4558,4564,4573,4579,4585,4588,459$ $7,4603,4606,4609,4630,4633,4645,4648,4654,4660,4669,4678,4684,4687,4690,4696,4702,4714,47$ $17,4720,4735,4738,4741,4744,4747,4753,4759,4768,4771,4774,4777,4783,4786,479$ $5,4801,4810,4822,4828,4834,4837,4843,4846,4855,4858,4864,4867,4873,4876,4882,4$ $885,4906,4909,4924,4942,4957,4960,4966,4975,4990,4999]$ 
$160=$

$[16,22,61,106,160,202,220,601,610,1006,1060,1345,1354,1435,1453,1534,1543,1600,2002,2020,2$ $200,2557,2575,2755,3145,3154,3415,3451,3514,3541,3559,3595,3955,4135,4153,4315,4351,4513$, $4531,4888]$

$\mathbf{4 0 7}=$

$[47,74,77,89,98,407,449,470,494,578,587,668,686,704,707,740,758,770,785,788,809,857,866,875$, $878,887,890,908,944,980,1124,1139,1142,1148,1157,1175,1178,1184,1187,1193,1214,1241,1319,1$ $367,1376,1391,1412,1418,1421,1481,1517,1559,1571,1589,1595,1598,1637,1673,1688,1715,1718$, 17

$36,1751,1763,1781,1814,1817,1841,1859,1868,1871,1886,1895,1913,1931,1955,1958,1985,2114,2$ $141,2249,2294,2333,2369,2378,2387,2396,2411,2429,2477,2492,2558,2585,2588,2639,2693,2738$, $2747,2774,2783,2837,2855,2858,2873,2885,2924,2936,2942,2963,3119,3167,3176,3191,3233,326$ $9,3278,3287,3296,3323,3332,3359,3377,3395,3539,3593,3617,3629,3671,3692,3716,3728,3737,37$ $61,3773,3782,3827,3872,3911,3926,3935,3953,3962,4007,4049,4070,4094,4112,4118,4121,4181,4$ $211,4229,4277,4292,4409,4448,4484,4490,4577,4700,4727,4757,4772,4775,4811,4844,4889,4898$, $4904,4922,4940,4988]$

$\mathbf{5 5}=$

$[55,505,550]$

$\mathbf{2 1 7}=$

$[79,97,115,127,151,172,217,271,388,511,709,712,721,790,838,883,907,970,1015,1027,1051,172,1$ $105,1150,1207,1270,1336,1363,1378,1387,1501,1510,1588,1633,1678,1687,1702,1720,1738$, $1768,1783,1786,1837,1858,1867,1873,1876,1885,2017,2071,2107,2170,2377,2446,2458,2464,248$ $5,2548,2584,2644,2701,2710,2737,2773,2845,2854,3088,3136,3163,3178,3187,3277,3316,3361,35$ $77,3613,3631,3718,3727,3757,3772,3775,3781,3808,3817,3871,3880,4246,4258,4264,4285,4426,4$ $462,4528,4582,4624,4642,4666,4699,4825,4852,4969,4996]$

$133=$

$[133,313,331,679,697,769,796,967,976,1033,1288,1303,1330,1828,1882,1999,2188,2818,2881,301$ $3,3031,3103,3130,3301,3310,3799,3979,3997]$

$352=$

$[229,235,238,253,283,292,325,328,352,382,445,454,457,475,523,532,544,547,574,745,754,823,83$ $2,922,2029,2035,2038,2053,2083,2092,2209,2290,2305,2308,2338,2350,2380,2383,2503,2530,280$ $3,2830,2833,2902,2920,3025,3028,3052,3082,3205,3208,3238,3250,3280,3283,3328,3382,3466,35$ $02,3520,3646,3664,3802,3820,3823,3832,4045,4054,4057,4075,4366,4405,4450,4504,4507,4540,4$ $570,4636,4663,4705,4750]$

$244=$

$[244,424,442,2044,2347,2374,2404,2437,2440,2473,2734,2743,3247,3274,3337,3373,3427,3472,3$ $724,3733,3742,4024,4042,4204,4237,4240,4273,4327,4372,4402,4420,4723,4732]$

371=

$[2,5,8,11,14,17,20,23,26,29,32,35,38,41,44,50,53,56,59,62,65,68,71,80,83,86,92,95,101,104,107,11$ $0,113,116,119,122,125,128,131,134,137,140,143,146,149,152,155,158,161,164,167,170,173,176,17$ $9,182,185,188,191,194,197,200,203,206,209,212,215,218,221,224,227,230,233,236,239,242,245,2$ $48,251,254,257,260,263,266,269,272,275,278,281,284,287,290,293,296,299,302,305,308,311,314$, $317,320,323,326,329,332,335,338,341,344,347,350,353,356,359,362,365,368,371,374,377,380,383$ ,386,389,392,395,398,401,404,410,413,416,419,422,425,428,431,434,437,440,443,446,452,455,45 $8,461,464,467,473,476,479,482,485,488,491,497,500,503,506,509,512,515,518,521,524,527,530,5$ 
$33,536,539,542,545,548,551,554,557,560,563,566,569,572,575,581,584,590,593,596,599,602,605$, $608,611,614,617,620,623,626,629,632,635,638,641,644,647,650,653,656,659,662,665,671,674,677$ ,680,683,689,692,695,698,701,710,713,716,719,722,725,728,731,734,737,

$743,746,749,752,755,761,764,767,773,776,779,782,791,794,797,800,803,806,812,815,818,821,824$ $, 827,830,833,836,839,842,845,848,851,854,860,863,869,872,881,884,893,896,899,902,905,911,91$ $4,917,920,923,926,929,932,935,938,941,947,950,953,956,959,962,965,968,971,974,977,983,986,9$ $89,992,995,998,1001,1004,1007,1010,1013,1016,1019,1022,1025,1028,1031,1034,1037,1040,1043$ ,1046,1049,1052,1055,1058,1061,1064,1067,1070,1073,1076,1079,1082,1085,1088,1091,1094,109 $7,1100,1103,1106,1109,1112,1115,1118,1121,1127,1130,1133,1136,1145,1151,1154,1160,1163,116$ $6,1169,1172,1181,1190,1196,1199,1202,1205,1208,1211,1217,1220,1223,1226,1229,1232,1235,12$ $38,1244,1247,1250,1253,1256,1259,1262,1265,1268,1271,1274,1277,1280,1283,1286,1289,1292,1$ $295,1298,1301,1304,1307,1310,1313,1316,1322,1325,1328,1331,1334,1337,1340,1343,1346,1349$, $1352,1355,1358,1361,1364,1370,1373,1379,1382,1385,1388,1394,1397,1400,1403,1406,1409,141$ $5,1424,1427,1430,1433,1436,1439,1442,1445,1448,1451,1454,1457,1460,1463,1466,1469,1472,14$ $75,1478,1484,1487,1490,1493,1496,1499,1502,1505,1508,1511,1514,1520,1523,1526,1529,1532,1$ $535,1538,1541,1544,1547,1550,1553,1556,1562,1565,1568,1574,1577,1580,1583,1586,1592,1601$, $1604,1607,1610,1613,1616,1619,1622,1625,1628,1631,1634,1640,1643,1646,1649,1652,1655,165$ $8,1661,1664,1667,1670,1676,1679,1682,1685,1691,1694,1697,1700,1703,1706,1709,1712,1721,17$ $24,1727,1730,1733,1739,1742,1745,1748,1754,1757,1760,1766,1769,1772,1775,1778,1784,1787,1$ $790,1793,1796,179$

$9,1802,1805,1808,1811,1820,1823,1826,1829,1832,1835,1838,1844,1847,1850,1853,1856,1862,18$ $65,1874,1877,1880,1883,1889,1892,1898,1901,1904,1907,1910,1916,1919,1922,1925,1928,1934,1$ 937,1940,1943,1946,1949,1952,1961,1964,1967,1970,1973,1976,1979,1982,1988,1991,1994,1997, 2000,2003,2006,2009,2012,2015,2018,2021,2024,2027,2030,2033,2036,2039,2042,2045,2048,205 $1,2054,2057,2060,2063,2066,2069,2072,2075,2078,2081,2084,2087,2090,2093,2096,2099,2102,21$ $05,2108,2111,2117,2120,2123,2126,2129,2132,2135,2138,2144,2147,2150,2153,2156,2159,2162,2$ $165,2168,2171,2174,2177,2180,2183,2186,2189,2192,2195,2198,2201,2204,2207,2210,2213,2216$, $2219,2222,2225,2228,2231,2234,2237,2240,2243,2246,2252,2255,2258,2261,2264,2267,2270,227$ $3,2276,2279,2282,2285,2288,2291,2297,2300,2303,2306,2309,2312,2315,2318,2321,2324,2327,23$ $30,2336,2339,2342,2345,2348,2351,2354,2357,2360,2363,2366,2372,2375,2381,2384,2390,2393,2$ $399,2402,2405,2408,2414,2417,2420,2423,2426,2432,2435,2438,2441,2444,2447,2450,2453,2456$, $2459,2462,2465,2468,2471,2474,2480,2483,2486,2489,2495,2498,2501,2504,2507,2510,2513,251$ $6,2519,2522,2525,2528,2531,2534,2537,2540,2543,2546,2549,2552,2555,2561,2564,2567,2570,25$ $73,2576,2579,2582,2591,2594,2597,2600,2603,2606,2609,2612,2615,2618,2621,2624,2627$, $2630,2633,2636,2642,2645,2648,2651,2654,2657,2660,2663,2666,2669,2672,2675,2678,2681,268$ $4,2687,2690,2696,2699,2702,2705,2708,2711,2714,2717,2720,2723,2726,2729,2732,2735,2741,27$ $44,2750,2753,2756,2759,2762,2765,2768,2771,2777,2780,2786,2789,2792,2795,2798,2801,2804,2$ $807,2810,2813,2816,2819,2822,2825,2828,2831,2834,2840,2843,2846,2849,2852,2861,2864,2867$, $2870,2876,2879,2882,2888,2891,2894,2897,2900,2903,2906,2909,2912,2915,2918,2921,2927,293$ $0,2933,2939,2945,2948,2951,2954,2957,2960,2966,2969,2972,2975,2978,2981,2984,2987,2990,29$ 93,2996,2999,3002,3005,3008,3011,3014,3017,3020,3023,3026,3029,3032,3035,3038,3041,3044,3 047,3050,3053,3056,3059,3062,3065,3068,3071,3074,3077,3080,3083,3086,3089,3092,3095,3098, $3101,3104,3107,3110,3113,3116,3122,3125,3128,3131,3134,3137,3140,3143,3146,3149,3152,3155$ ,3158,3161,3164,3170,3173,3179,3182,3185,3188,3194,3197,3200,3203,3206,3209,3212,3215,321 $8,3221,3224,3227,3230,3236,3239,3242,3245,3248,3251,3254,3257,3260,3263,3266,3272,3275,32$ $81,3284,3290,3293,3299,3302,3305,3308,3311,3314,3317,3320,3326,3329,3335,3338,3341,3344,3$ $347,3350,3353,3356,3362,3365,3368,3371,3374,3380,3383,3386,3389,3392,3398,3401,3404,3407$, $3410,3413,3416,3419,3422,3425,3428,3431,3434,3437,3440,3443,3446,3449,3452,3455,3458,346$ $1,3464,3467,3470,3473,3476,3479,3482,3485,3488,3491,3494,3497,3500,3503,3506,3509,3512,35$ $15,3518,3521,3524,3527,3530,3533,3536,3542,3545,3548,3551,3554,3557,3560,3563,3566,3569,3$ $572,3575,3578,3581,3584,3587,3590,3596,3599,3602,3605,3608,3611,3614,3620,3623,3626,3632$, $3635,3638,3641,3644$, 
$3647,3650,3653,3656,3659,3662,3665,3668,3674,3677,3680,3683,3686,3689,3695,3698,3701,370$ $4,3707,3710,3713,3719,3722,3725,3731,3734,3740,3743,3746,3749,3752,3755,3758,3764,3767,37$ $70,3776,3779,3785,3788,3791,3794,3797,3800,3803,3806,3809,3812,3815,3818,3821,3824,3830,3$ $833,3836,3839,3842,3845,3848,3851,3854,3857,3860,3863,3866,3869,3875,3878,3881,3884,3887$, $3890,3893,3896,3899,3902,3905,3908,3914,3917,3920,3923,3929,3932,3938,3941,3944,3947,395$ $0,3956,3959,3965,3968,3971,3974,3977,3980,3983,3986,3989,3992,3995,3998,4001,4004,4010,40$ $13,4016,4019,4022,4025,4028,4031,4034,4037,4040,4043,4046,4052,4055,4058,4061,4064,4067,4$ $073,4076,4079,4082,4085,4088,4091,4097,4100,4103,4106,4109,4115,4124,4127,4130,4133,4136$, $4139,4142,4145,4148,4151,4154,4157,4160,4163,4166,4169,4172,4175,4178,4184,4187,4190,419$ $3,4196,4199,4202,4205,4208,4214,4217,4220,4223,4226,4232,4235,4238,4241,4244,4247,4250,42$ $53,4256,4259,4262,4265,4268,4271,4274,4280,4283,4286,4289,4295,4298,4301,4304,4307,4310,4$ $313,4316,4319,4322,4325,4328,4331,4334,4337,4340,4343,4346,4349,4352,4355,4358,4361,4364$, $4367,4370,4373,4376,4379,4382,4385,4388,4391,4394,4397,4400,4403,4406,4412,4415,4418,442$ $1,4424,4427,4430,4433,4436,4439,4442,4445,4451,4454,4457,4460,4463,4466,4469,4472,4475,44$ $78,4481,4487,4493,4496,4499,4502,4505,4508,4511,4514,4517,4520,4523,4526,4529,4532,4535,4$ $538,4541,4544,4547,4550,4553,4556,4559,4562,4565,4568,4571,4574,4580,4583,4586,4589,4592$, $4595,4598,4601,4604,4607,4610,4613,4616,4619,4622,4625,4628,4631,4634,4637,4640,4643,464$ $6,4649,4652,4655,4658,4661,4664,4667,4670,4673,4676,4679,4682,4685,4688,4691,4694,4697,47$ $03,4706,4709,4712,4715,4718,4721,4724,4730,4733,4736,4739,4742,4745,4748,4751,4754,4760,4$ $763,4766,4769,4778,4781,4784,4787,4790,4793,4796,4799,4802,4805,4808,4814,4817,4820,4823$, $4826,4829,4832,4835,4838,4841,4847,4850,4853,4856,4859,4862,4865,4868,4871,4874,4877,488$ $0,4883,4886,4892,4895,4901,4907,4910,4913,4916,4919,4925,4928,4931,4934,4937,4943,4946,49$ 49,4952,4955,4958,4961,4964,4967,4970,4973,4976,4979,4982,4985,4991,4994,4997]

\section{References}

[1] http://scienceasia.asia/index.php/amp/article/view/23/6

[2] http://scienceasia.asia/index.php/amp/article/view/24/7

[3] http://www.ijmsea.com/admin/docs/1379247228yes-3.pdf

[4] www.drgandhi.info

[5] http://en.wikipedia.org/wiki/Miraculous_catch_of_fish

[6] Elementary Theory of Numbers: Second English Edition (edited by A. Schinzel) by W. Sierpinski, Pwn-Polish Scientific publishers, 1988. 